\title{
Content and Network-Aware Multicast Over Wireless Networks
}

\author{
Chamitha de Alwis, H. Kodikara Arachchi and Anil Fernando \\ I-Lab, Centre for Vision, Speech and Signal Processing \\ University of Surrey \\ Guildford, United Kingdom \\ \{C.Dealwis, H.Kodikaraarachchi, W.Fernando\}@ surrey.ac.uk
}

\begin{abstract}
This paper proposes content and network-aware redundancy allocation algorithms for channel coding and network coding to optimally deliver data and video multicast services over error prone wireless mesh networks. Each network node allocates redundancies for channel coding and network coding taking in to account the content properties, channel bandwidth and channel status to improve the end-to-end performance of data and video multicast applications. For data multicast applications, redundancies are allocated at each network node in such a way that the total amount of redundant bits transmitted is minimised. As for video multicast applications, redundancies are allocated considering the priority of video packets such that the probability of delivering high priority video packets is increased. This not only ensures the continuous playback of a video but also increases the received video quality. Simulation results for bandwidth sensitive data multicast applications exhibit up to $10 \times$ reduction of the required amount of redundant bits compared to reference schemes to achieve a $100 \%$ packet delivery ratio. Similarly, for delay sensitive video multicast applications, simulation results exhibit up to $3.5 \mathrm{~dB}$ PSNR gains in the received video quality.
\end{abstract}

Keywords- network coding; content and network-aware redundancy allocation; wireless mesh networks

\section{INTRODUCTION}

Wireless mesh networks (WMNs) are increasingly being deployed to provide cost efficient and low maintenance internet access [1]-[4]. However, due to fading, wireless links in WMNs exhibit extremely high packet loss rates. For instance, half of the links in Roofnet [1] exhibit over 30\% packet loss rates. Therefore, improving the resilience of WMNs against packet losses is one of the key themes in wireless communication research [3]-[6]. On the other hand, there is a growing popularity of high volume video multicast services such as internet TV, live streaming of sports events, etc. Furthermore, statistical predictions indicate up to a fivefold increase in the usage of internet video and internet TV by 2017 [7]. Hence, it is also a significant challenge to efficiently deliver video at a high quality across packet loss prone WMNs.

Techniques of using network coding [8] based multicast protocols are proved to increase the throughput and robustness of data multicast applications across WMNs, compared to traditional routing schemes [3], [4]. The network coding algorithms proposed in [3] and [4] allow network nodes to sense forward links and estimate the number of coded packets they should transmit in order to increase the throughput and reliability of data multicast applications. However, due to the inefficient redundancy allocation of these algorithms, they are not optimal for bandwidth sensitive data multicast applications. Furthermore, it should be noted that in video multicast applications, it is essential to deliver at least the most important packets in a timely manner to provide an uninterrupted video service at an acceptable quality. Several network coding algorithms, including [9], propose to sense the network status and adaptively transmit scalable video layers to increase the received video quality over traditional routing schemes. However, since state-of-the-art network coding algorithms for video multicast applications are not capable of adaptively allocating redundancies at each network node in a content and network-aware fashion, they are not optimal to multicast video across WMNs.

In response, this paper proposes intelligent and distributed content and network-aware algorithms to allocate redundancies for network coding to improve the performance of data and video multicast applications across WMNs. An overview of the proposed algorithms is illustrated in Figure 1. Accordingly, using the proposed algorithms, each network node considers the channel bandwidth and channel status to allocate the appropriate amount of redundancies such that the number of redundant bits to be transmitted is minimised for data multicast applications. As for video multicast applications, content properties are also considered along with channel properties to improve the quality of video multicast applications. The rest of the paper is organised as follows. Section II summarises the work available in the literature relevant to the presented work. Section III describes the proposed algorithms. Section IV presents the performance evaluation of the proposed algorithm, comparing against reference schemes. Section V concludes the paper.

\section{RELATED WORK}

Network coding extends information coding to intermediate network nodes in order to increase the network throughput and robustness against bit errors and packet losses [8]. Random linear network coding [10]-[13] extends network coding to be used in random network structures by means of transmitting a set of coefficients updated at each node a packet passes through a network. Practical network coding [14] introduces the concept of performing random linear network coding for groups of packets called generations to further extend the 


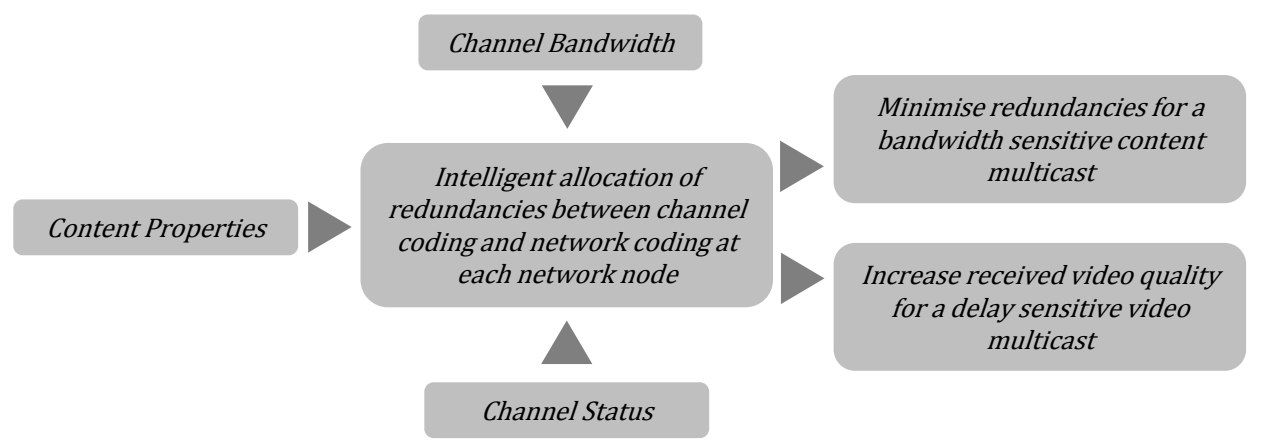

Figure 1. Overview of the proposed algorithm

usability of network coding in realistic networks with packet erasures, delays and topology changes. In addition, practical network coding allows transmitting redundant network coded packets as a Forward Error Correction (FEC) mechanism to increase the probability of decoding network coded packet generations amidst packet erasures. These redundant network coded packets perform FEC more efficiently than traditional Automatic Repeat reQuest (ARQ) based schemes to combat packet erasures [15]. Combining random linear network coding together with opportunistic routing, MORE [3] exploits the opportunism inherent in the wireless medium. In doing so, the throughput is significantly increased compared to traditional routing and prior work on opportunistic routing. Pacifier [3] further improves the throughput of a data multicast across a WMN by integrating tree-based opportunistic routing, intraflow network coding, source-rate limiting and round-robin batching. Bandwidth sensitive data multicast applications, such as software updates required by many nodes across a network, require a $100 \%$ packet delivery ratio. For these kind of applications, transmitting coded packets until receivers acknowledge the receiving of sufficient coded packets to decode a network coded packet generation, such as in [3], [4] is acceptable. For this reason, less attention is paid towards adding redundancies such that the FEC capability of channel coding and network coding is enhanced considering the network bandwidth and channel status. As a result, the number of redundant bits transmitted is not optimally minimised to maximise the throughput and energy efficiency of a network.

On the other hand, a delay sensitive video multicast such as internet television requires the timely delivery of video packets to ensure continuous video playback. Hence, it is not possible for a source node to delay the transmission of coded packets until all receivers acknowledge the reception of sufficient coded packets to decode the preceding network coded packet generation. Therefore, video packets are transmitted with redundancies added at the source node as a measure of FEC. In addition, a video multicast does not essentially require a $100 \%$ packet delivery rate as video decoders are capable of concealing missing video packets. Moreover, depending on the content, different video packets have a different impact in recovering the original video sequence. For this reason, the Distributed Robust Optimisation for Scalable Video Multirate Multicast (DROSVMM) algorithm proposed in [9] uses the information from all network nodes to jointly optimise the overall video quality for a scalable video multirate multicast using network coding. In doing so, each scalable layer is tailored in an incremental order to find jointly optimal multicast paths and associated rates. The usage of network coding in the above algorithm increases the network throughput and enhances the robustness of a video transmission against packet losses. Nevertheless, the above algorithms allocate redundancies at the source node and the redundancy rate is kept constant throughout the multicast. Furthermore, the redundancy allocation in this algorithm is not optimal because it doesn't consider either the priority levels of scalability layers of a video or the status of individual links.

Addressing these issues, we develop a content and networkaware algorithm to allocate redundancies for channel coding and network coding to optimise the multicast of bandwidth and delay sensitive content over error prone WMNs.

\section{Methodology}

The proposed algorithm initiates by distinguishing a multicast as either bandwidth sensitive or delay sensitive video. As for delay sensitive video multicast, the proposed algorithm further identifies the priority of video packets based on the scalable video layer. Then, considering the channel status and channel bandwidth, the proposed algorithm attempts to use available network resources efficiently in order to,

1. Minimise the total amount of redundancies transmitted in a bandwidth sensitive data multicast.

2. Ensure the continuous playback of video while increasing the received video quality in a delay sensitive video multicast.

\section{A. Data Multicast Applications}

The source node builds a shortest-ETX multicast tree [16] based on loss rate measurements, similar to [4] in order to connect the source to all receivers in a network. Next, the source node selects $n$ packets, where each packet is a set of $\sigma$ symbols from a Galois Field of size $2^{F}$. These $n$ packets belong to the network coding generation $G$. Let $i$ be either the source node or an intermediate network node and $j_{1}, j_{2}, \ldots, j_{v}$ be $v$ child nodes of node $i$, in the considered network. $\pi_{i}$ denotes the coefficient rank of the received coded packets by node $i$, from its ancestor nodes. Node $i$ should make sure that all child nodes receive the same amount of information as it has. Hence, in case of no packet errors, the number of coded packets that node $i$ should transmit by performing random linear network coding, $z_{i}$, is, 


$$
z_{i}=\pi_{i}-\min \left(\pi_{j_{1}}, \pi_{j_{2}}, \ldots, \pi_{j_{v}}\right)
$$

However, since wireless links exhibit packet errors, redundancies should be added to ensure the delivery of at least $z_{i}$ number of coded packets by all $v$ child nodes. To achieve this with the minimal amount of redundancies, we propose to ensure the delivery of at least $z_{i}$ number of coded packets by all $v$ child nodes at the probability of $r_{p}$. A value for $r_{p}$ is determined empirically such that the total number of redundant bits transmitted is minimised, as discussed in Section IV.

Let the code rate of channel coding in the physical layer be $C_{c h}$ and $\gamma$ be the average signal to noise ratio. Similar to the assumptions followed in [17], for a capacity achieving channel coding scheme such as Turbo and low density parity check coding (LDPC), the probability of correctly transmitting a packet, $q$, across a Nakagami-m fading channel is,

$$
q=\sum_{k=0}^{m-1} \frac{1}{k !}(m \alpha)^{k} e^{-m \alpha}
$$

where, $\alpha=\frac{\left(2^{C} c h-1\right)}{\gamma}$.

$C_{c h_{i}}$ is the channel code rate. It should also be noted that when $m=1$, the Nakagami-m channel reduces to a Rayleigh fading channel.

A mesh network consisting of wireless links exhibiting flatfading is considered. Furthermore, it is assumed that wireless receptions at different network nodes are independent. Let the average signal to noise ratio at child nodes $j_{1}, j_{2}, \ldots j_{v}$ be $\gamma_{j_{1}}, \gamma_{j_{2}}, \ldots \gamma_{j_{n}}$ respectively. Recall node i should make sure that all child nodes receive the same amount of information as it has. Hence redundancies are allocated for FEC considering the child node with the minimum signal to noise ratio, $\gamma_{c}$.

$$
\gamma_{c}=\min \left(\gamma_{j_{1}}, \gamma_{j_{2}}, \ldots \gamma_{j_{n}}\right)
$$

Using the Binomial distribution, the probability of correctly transmitting at least $z_{i}$ packets, $r$ is calculated as,

$$
r=\sum_{l=z_{i}}^{y_{i}}\left(\begin{array}{c}
y_{i} \\
l
\end{array}\right) q_{c}{ }^{i}\left(1-q_{c}\right)^{y_{i}-l}
$$

where, $y_{i}\left(\geq z_{i}\right)$ is the total number of coded packets (including redundant coded packets, if necessary) generated at node $i . q_{c}$ is the probability of correctly transmitting a packet when the average signal to noise ratio is $\gamma_{c}$, calculated using (2).

Since random linear network coding requires the transmission of $\mathrm{n}$ coefficients per each coded packet, the network code rate, $C_{n w}$ can be calculate as.

$$
C_{n w}=\frac{y_{i}}{z_{i}} \cdot \frac{p}{(n+p)}
$$

Where $\frac{p}{(n+p)}$ is the network coding overhead. Thus, the total code rate of node $i,\left(C_{t_{i}}\right)$ is,

$$
C_{t_{i}}=C_{c h_{i}} \cdot C_{n w_{i}}
$$

Let the optimal values for $C_{c h_{i}}$ and $y_{i}$ such that the number of redundant bits transmitted is minimum be $\dot{C}_{c h_{i}}$ and $\dot{y}_{i}$ respectively. $\dot{C}_{c h_{i}}$ and $\dot{y}_{i}$ are calculated when $C_{t_{i}}$ is maximum,

$$
\operatorname{Max}\left(C_{t_{i}}\right)
$$

Such that,

$$
r \geq r_{p}
$$

It should be noted that $\dot{C}_{c h_{i}}$ and $\dot{y}_{i}$ depicts the optimal distribution of redundancies between channel coding and network coding for the prevailing link condition. However, in case if the channel bandwidth is limited, the proposed method transmits the maximum possible amount of coded packets.

The source node transmits packets until receivers acknowledge the sufficient reception of coded packets to decode a network coded packet generation. Upon receiving the acknowledgement from all receivers, the source multicasts the consecutive network coded packet generation. Once coded packets from the new generation is received, intermediate nodes flush packets belonging to the previous generation from the buffer.

\section{B. Video Multicast Applications}

The proposed algorithm for a delay sensitive video multicast is overviewed in Figure 2. Accordingly, each network node considers the priority of incoming video packets and network parameters such as channel status and channel bandwidth of child nodes. Then, redundancies are allocated for channel coding and network coding, increasing the probability of delivering at least the high priority scalable video layers. Similar to a bandwidth sensitive data multicast, the proposed algorithm for delay sensitive video multicast applications initiates by building a shortest-ETX multicast tree to connect the source node to all of the receivers. Subsequently, video packets are linearly coded at the source node, as explained below.

Consider the transmission of a GOP belonging to a scalable video stream containing the base layer and $k-1$ enhancement layers. Let each layer have $n_{1}, n_{2}, \ldots n_{k}$ number of packets with each packet having $\sigma$ symbols from a Galois Field of size $2^{F}$. Packets belonging to the base layer and individual enhancement layers are allocated to $k$ distinct network coded packet generations, $\eta,(\eta+1), \ldots,(\eta+k-1)$. In addition, each coded packet belonging to $k$ scalable video layers are flagged with the priority of the scalable video layer using the content priority class, as illustrated in Figure 3 . This will allow network nodes to identify the scalable video layer of the video data contained in an incoming coded packet. Accordingly, coded packets belonging to the base layer of a video has a content priority class of zero (highest priority) whereas coded packets belonging to the $e^{\text {th }}$ enhancement video layer has a content priority class of $e$. After transmitting coded packets belonging to $k$ generations, the source node begins transmitting coded packets from the consecutive GOP. It should be noted that there is no waiting for receiver acknowledgement in case of a video multicast unlike in a bandwidth sensitive data multicast in order to minimise transmission delay and jitter. 


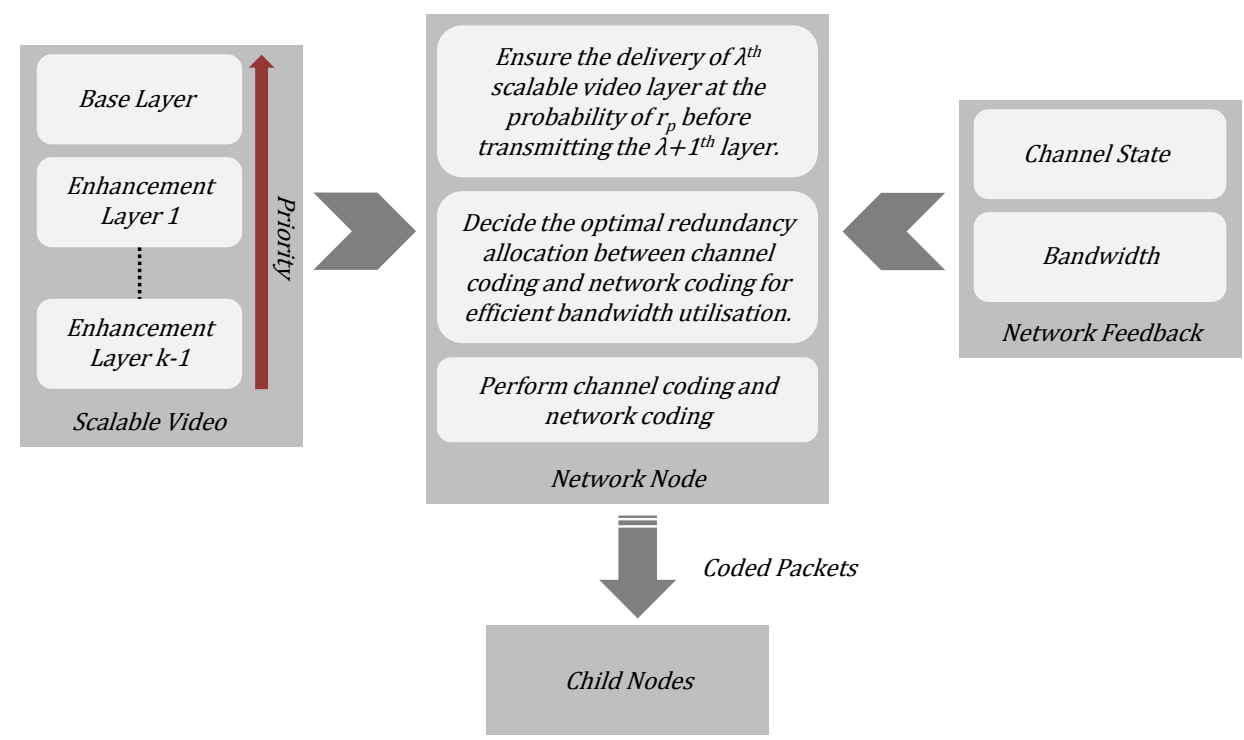

Figure 2. Overview of the proposed algorithm for video transmission

Hereafter, operations performed at the source node and relaying nodes are similar, as explained below.

Consider a node $i$, which may either be the source node or a relay node in the network. The node $i$ is required to transmit $z_{i g}$ number of coded packets from the generation $g$ similar to the case of a bandwidth sensitive data transmission. $z_{i g}$ can be calculated using (1). Next, considering the channel status, node $i$ calculates the optimum number of coded packets $y_{i g}$ and the optimal channel code rate, $\dot{C}_{c h_{i}, g}$ for the generation $g . y_{i g}$ and $\dot{C}_{c h_{i}, g}$ are determined in such a way that the total code rate, $C_{t_{i}}$ is maximised while $r \geq r_{p} . C_{t_{i}}$ is calculated using (6). A value for $r_{p}$ is determined empirically such that the received video quality is maximised for a video multicast, as elaborated in Section IV.

Let the bandwidth required to transmit $y_{i g}$ number of coded packets be $b_{i}$. Therefore, if the channel bandwidth is $d_{i}$, the requirement to transmit all scalable video layers with the optimal number of coded packets is,

$$
\sum_{g=1}^{k} b_{i_{g}} \leq d_{i}
$$

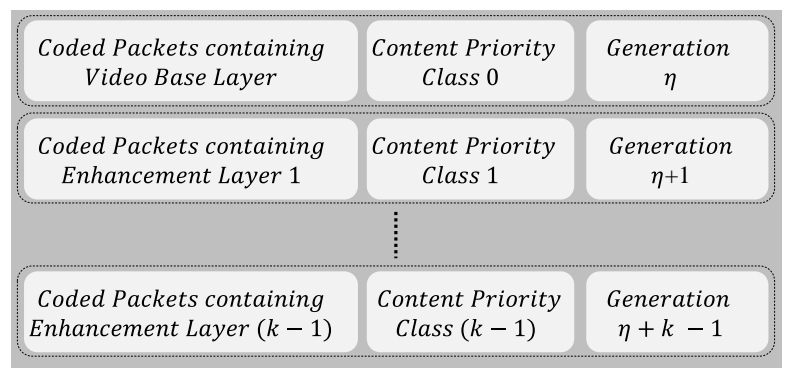

Figure 3. Assignment of content priority classes for coded packets from scalable video layers.
However, in case (8) is not satisfied, node i transmits coded packet belonging to $u<k$ generations, considering the priority of scalable video layers. $u$ is calculated such that,

$$
\sum_{g=1}^{u} b_{i_{g}} \leq d_{i}<\sum_{g=1}^{u+1} b_{i_{g}}
$$

Additionally, the remaining bandwidth (i.e., $d_{i}-\sum_{g=1}^{u} b_{i g}$ ) is used to transmit the maximum possible amount of packets from generation $u+1$. These might be useful in case if network nodes and receivers in the downstream receive additional innovative packets by means of opportunistic listening.

\section{PERFORMANCE EVALUATION}

\section{A. Simulation Setup}

The performance of the proposed method is evaluated by means of performing an extensive simulation study on a wireless mesh network with 50 static nodes randomly placed in an area of $1000 m \times 1000 m$. The available bandwidth of each link is considered to be different from each other. All wireless links are Rayleigh fading channels. The channel coding scheme considered in this paper is LDPC with discrete code rates of $\frac{7}{8}, \frac{5}{6}, \frac{3}{4}$ and $\frac{2}{3}$. The Galois Field size for network coding is $2^{8}$, which is sufficiently large enough for practical applications [14]. Each coded packet contains $s=1400$ symbols from the considered Galois Field. The network coding coefficient vectors are compressed using the algorithm proposed in [18]. Each video frame is divided in to slices of 1250 bytes in order to be accommodated along with the compressed network coding vectors in a network coded packet with 1400 symbols from the considered Galois Field.

For data multicast simulations, a scenario in which the source node transmits a $1.35 \mathrm{MB}$ file is considered. Similarly, for delay sensitive video multicast simulations, high definition video sequences encoded using the scalable extension of H.264/AVC in the JSVM encoder are transmitted. Per each 
simulation, over 3000 video frames are transmitted across the simulated network. The considered video sequences are of 1920 x 1080 pixel resolution. The base layer and two enhancement layers are encoded with QPs 35, 30 and 25, respectively. These values are selected such that each scalable layer has a distinctive difference in bit-rates and quality.

\section{B. Evaluation Metrics}

Following are the metrics used in evaluating the performance of the proposed algorithms compared to the reference schemes.

1) Data Multicast Applications: The objective of a bandwidth sensitive data multicast application is to achieve a $100 \%$ packet delivery ratio by means of either FEC or ARQ by transmitting minimal number of redundant bits. It should also be noted that the proposed method allocates redundancies for channel coding and network coding at each network node independently. Acordingly, the following matrix is considered to evaluate the performance of the proposed method.

$$
\begin{gathered}
\begin{array}{l}
\text { Number of bits transmitted per node per source bit } \\
\sum_{\forall \text { network nodes }} \text { total number of bits transmitted }
\end{array} \\
\begin{array}{c}
\text { number of network nodes } \\
\text { in the multicast tree }
\end{array} \times \begin{array}{c}
\text { original file } \\
\text { size in bits }
\end{array}
\end{gathered}
$$

2) Video multicast Applications: Unlike in a data multicast application, the objective of a delay sensitive video multicast application is to deliver video at a high quality. In order to achieve this, the proposed method independently allocates channel coding and network coding redundancies at each network node such that high priority video packets are delivered at a high probability. Hence, the following metric is used to evaluate the performance of the proposed algorithm for video multicast applications.

$$
\begin{aligned}
& \text { Average Received Video Quality } \\
& =\frac{\sum_{\forall \text { receivers }} \sum_{\forall \text { video frames }} \text { PSNR of received video frame }}{\text { number of receivers } \times \text { transmitted videoframes }}
\end{aligned}
$$

\section{Simulation Results}

1) Data Multicast Applications: Initial simulations are performed to determine an empirical value for $r_{p}$. Figure 4 illustrates the number of bits transmitted per node per source bit with the proposed algorithm vs. $r_{p}$ for average signal to noise ratio values of 5,10 and 15 . This shows that the number of bits transmitted per node per source bit gradually decreases with $r_{p}$ until a minimum and increases thereafter.

The above observations can be explained by considering (4) and (5). Accordingly, smaller the value of $r_{p}$ is, smaller the value of $C_{n w}$ becomes. Hence fewer redundancies are added per transmission. On the other hand, using a lower $r_{p}$ value results in a lower probability of accurately transmitting a coded packets from node to its child nodes. This in return

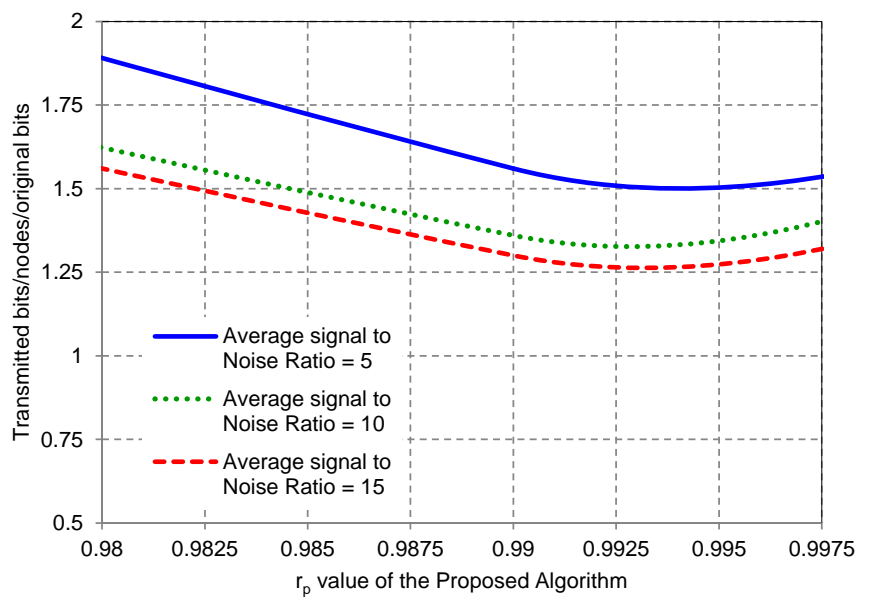

Figure 4. Transmitted bits/nodes/original bits vs. $\boldsymbol{r}_{\boldsymbol{p}}$ value of the proposed algorithm

reduces the end-to-end probability of successfully transmitting a coded packet from the source node to all receivers. Since the source keeps on transmitting coded packets until all the receivers acknowledge, a lower probability of successful transmission of a coded packet increases the number of retransmission attempts. Compromising the amount of redundancies added per transmission and the number of retransmission attempts, an empirical value for optimum $r_{p}$ can be determined in such a way that the total number of bits transmitted is minimised. Based on the experimental results presented in Figure 4, $r_{p}=0.9925$ is chosen as the optimum value. This value is used for subsequent experiments.

The subsequent set of experiments compare the performance of the proposed method against the reference scheme [4]. Since the considered channel code rates are not mentioned in [4], the reference technology is simulated for all considered channel code rates. Figure 5 illustrates the average number of bits transmitted vs. the average signal to noise ratio. Accordingly, it can be observed that the proposed algorithm can significantly reduce the average number of transmitted bits compared to the reference scheme. Unlike the proposed algorithm, [4] does not estimate the redundancies that should be allocated for channel coding and network coding to minimise the average number of transmitted bits. As a result, the performance of the proposed algorithm improves over the reference scheme. Furthermore, the end-to-end probability of successful transmission of a coded packet increases rapidly with the number of hops when the per-hop packet delivery probability is lower. However, since the proposed method ensures a high per-hop packet delivery probability, it is more scalable for the size of the network compared to the algorithm proposed in [4]. This argument is supported by the results presented in Figure 6, which illustrates the average number of bits transmitted vs. the number hops. 


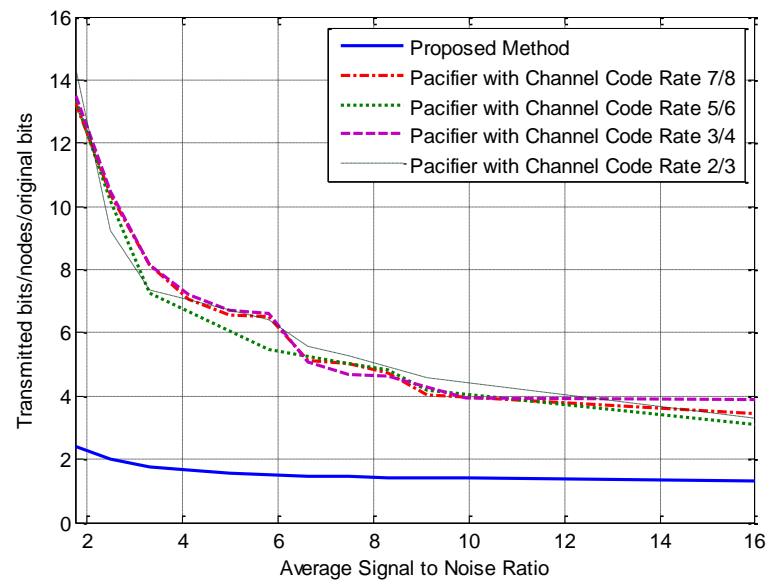

Figure 5. Transmitted bits/nodes/original bits vs. average signal to noise ratio.

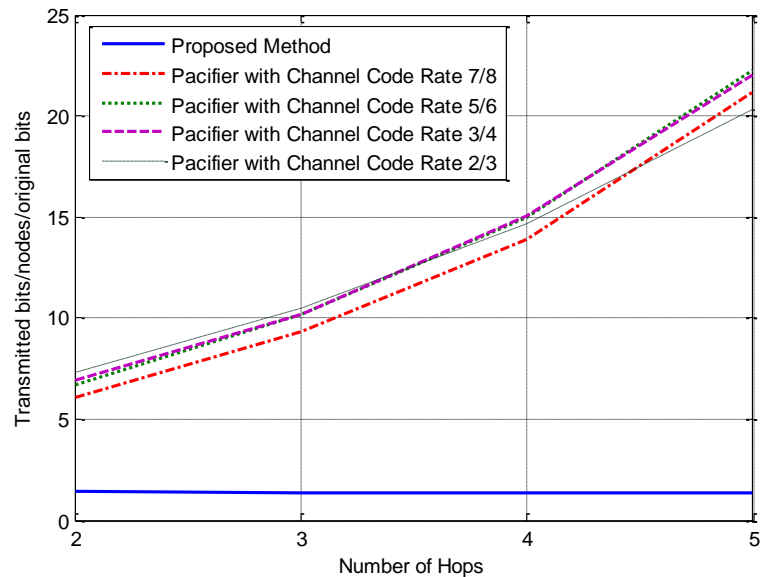

Figure 6. Transmitted bits/nodes/original bits vs. number of hops.

2) Video Multicast Applications: An extensive simulation study is performed to evaluate the performance of the proposed algorithm using a number of video sequences. For the demonstration purposes, results are presented for Basketball Drive and Cactus sequences due to space limitations.

Similar to the case of bandwidth sensitive data multicast, a suitable $r_{p}$ value for delay sensitive video multicast applications is determined empirically such that the average received video quality is maximised. Figure 7 and Figure 8 illustrate the average received video quality for the proposed method vs. $r_{p}$ for average signal to noise ratio values of 5,10 and 15 . The average received video quality for all the sequences over the simulated range of signal to noise ratios is maximised when $r_{p}=0.9999$. Hence this value is selected for further simulations presented in this section. It should be noted that the optimum $r_{p}$ value for delay sensitive video multicast applications is greater than that for bandwidth sensitive data multicast applications. This is because the only error correction mechanism applied for delay sensitive video multicast is FEC, as discussed in Section III.B.

The next set of experiments is performed in order to evaluate the performance of the proposed algorithm for video multicast applications. Results are compared against DROSVMM, which is a state-of-the-art reference technique to improve the performance of delay sensitive video multicast applications in wireless mesh networks [9]. Due to similar reasons discussed in Section IV.C.1, the reference technique [9] is simulated for all the considered channel code rates.

Figure 9 and Figure 10 illustrate the average received video quality obtained using the simulated algorithms against the average signal to noise ratio. These results indicate that the proposed technology consistently outperforms the reference technique. In the reference technique, channel and network code rates remain constant throughout the network regardless of the differences in network condition at different links. The proposed algorithm performs better since it allows individual network nodes to independently allocate redundancies for channel and network coding considering the content priority, channel condition and available bandwidth. Hence, the proposed algorithms are more suitable for delay sensitive video multicast applications, compared to reference schemes.

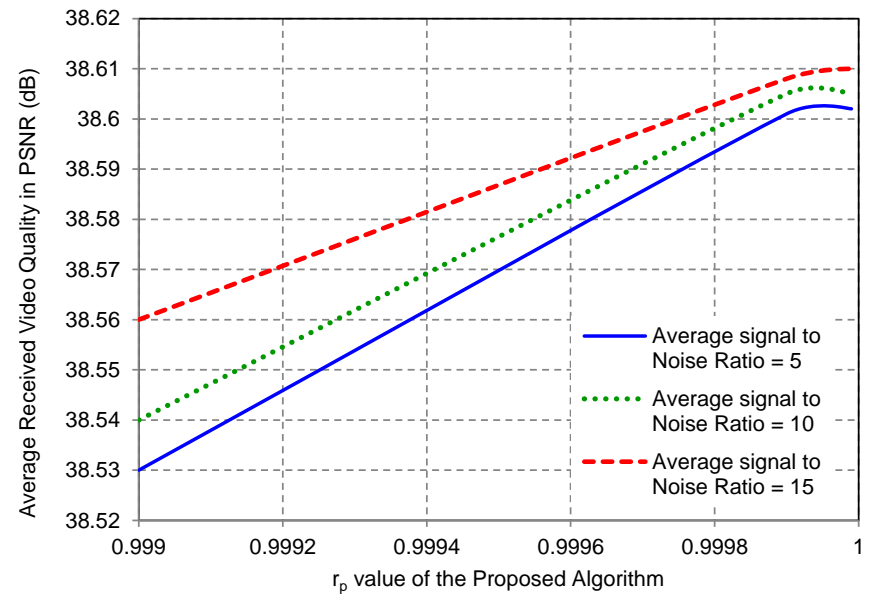

Figure 7. Average received video quality vs. $\boldsymbol{r}_{\boldsymbol{p}}$ value of the proposed algorithm for the Basketball Drive sequence.

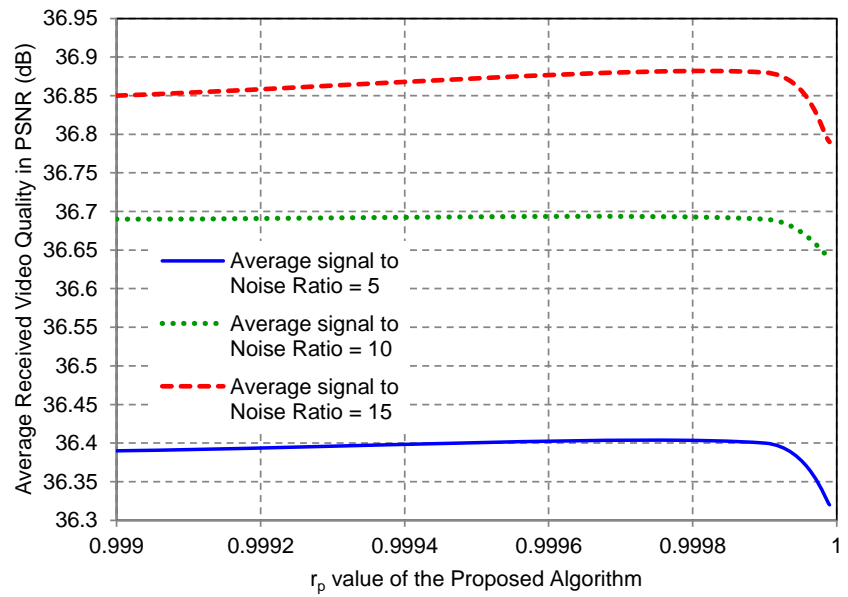

Figure 8 . Average received video quality vs. $\boldsymbol{r}_{\boldsymbol{p}}$ value of the proposed algorithm for the Cactus sequence. 


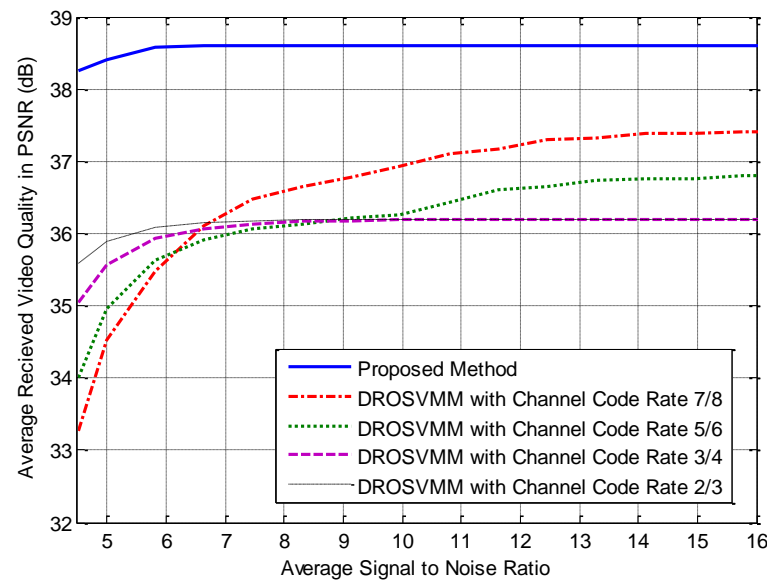

Figure 9. Average received video quality vs. average signal to noise ratio for the Basketball Drive sequence.

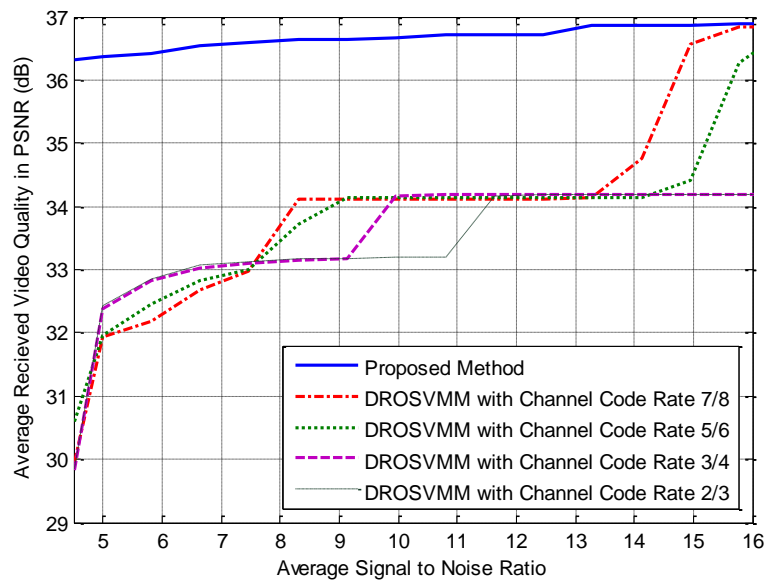

Figure 10. Average received video quality vs. average signal to noise ratio for the Cactus sequence.

\section{CONCLUSIONS}

This paper proposes a novel content and network-aware algorithm to intelligently distribute redundancies between channel coding and network coding at each network node. The objectives are to minimise the redundancies for bandwidth sensitive multicast applications and increase the received video quality for delay sensitive video multicast applications. Simulation results for bandwidth sensitive data multicast applications indicate up to $10 \times$ reduction in the number of redundant bits transmitted. Up to $3.5 \mathrm{~dB}$ gains in user received video quality is observed for delay sensitive video multicast applications. Hence the proposed method can be used to efficiently utilise wireless resources for multicast applications in packet loss prone WMNs.

What is more, the proposed algorithm for delay sensitive multicast applications is not only limited for scalable video transmission but can be explored for other forms of video transmission where video content can be classified in to multiple content priority classes. As a future work, the parameters of the proposed algorithm such as $r_{p}$ can be dynamically optimised for various network conditions and content types to further enhance the performance.

\section{REFERENCES}

[1] "MIT roofnet." [Online]. Available: http://www.pdos.lcs.mit.edu/roofnet.

[2] "Seattle wireless." [Online]. Available: http://www.seattlewireless.net.

[3] S. Chachulski, M. Jennings, S. Katti, and D. Katabi, "Trading structure for randomness in wireless opportunistic routing.," in SIGCOMM, 2007, pp. $169-180$.

[4] D. Koutsonikolas, Y. C. Hu, and C.-C. Wang, "Pacifier: highthroughput, reliable multicast without 'crying babies' in wireless mesh networks," IEEEACM Trans. Netw., vol. 20, no. 5, pp. 1375-1388, 2012.

[5] cisco, "The zettabyte era - trends and analysis." May-2013.

[6] D. Koutsonikolas and Y. C. Hu, "The case for FEC-based reliable multicast in wireless mesh networks," in IEEE/IFIP International Conference on Dependable Systems and Networks, 2007, pp. 491-501.

[7] L. Rizzo and L. Vicisano, "RMDP: an FEC-based reliable multicast protocol for wireless environments", Mobile Computing and Communications Review, vol. 2, 1998.

[8] R. Ahlswede, N. Cai, S.-Y. R. Li, and R. W. Yeung, "Network information flow," IEEE Trans. Inf. Theory, vol. 46, no. 4, pp. 12041216, 2000.

[9] C. Li, H. Xiong, J. Zou, and C. W. Chen, "Distributed robust optimization for scalable video multirate multicast over wireless networks," IEEE Trans. Circuits Syst. Video Technol., vol. 22, no. 6, pp. 943-957, 2012.

[10] T. Ho, R. Koetter, M. Medard, D. R. Karger, and M. Effros, "The benefits of coding over routing in a randomized setting," in IEEE International Symposium on Information Theory, 2003.

[11] T. Ho, M. Medard, R. Koetter, D. R. Karger, M. Effros, J. Shi, and B. Leong, "A Random Linear Network Coding Approach to Multicast," IEEE Trans. Inf. Theory, vol. 52, no. 10, pp. 4413-4430, 2006.

[12] T. Ho, B. Leong, R. Koetter, M. Medard, M. Effros, and D. R. Karger, "Byzantine modification detection in multicast networks using randomized network coding," in International Symposium on Information Theory, 2004.

[13] M. M. Desmond S. Lun, "On coding for reliable communication over packet networks," Phys. Commun., no. 1, pp. 3-20, 2005.

[14] P. A. Chou, Y. Wu, and K. Jain, "Practical network coding," in Annual Allerton Conference on Communication Control and Computing, 2003, vol. 41 , pp. $40-49$.

[15] D. Nguyen, T. Tran, T. Nguyen, and B. Bose, "Wireless broadcast using network coding," IEEE Trans. Veh. Technol., vol. 58, no. 2, pp. 914925, 2009.

[16] D. S. De Couto, D. Aguayo, J. Bicket, and R. Morris, "A highthroughput path metric for multi-hop wireless routing," Wirel. Netw., vol. 11, no. 4, pp. 419-434, 2005.

[17] C. R. Berger, S. Zhou, Y. Wen, P. Willett, and K. Pattipati, "Optimizing joint erasure and error correction coding for wireless packet transmissions," IEEE Trans. Wirel. Commun., vol. 7, no. 11, pp. 45864595, 2008.

[18] P. A. Chou, Y. Wu, and K. Jain, "Practical network coding," in Allerton Conference on Communication Control and Computing, 2003, vol. 41, pp. $40-49$.

[19] C. de Alwis, H. K. Arachchi, A. Fernando, and A. Kondoz, "Towards minimising the coefficient vector overhead in random linear network coding," in IEEE International Conference on Acoustics, Speech and Signal Processing, 2013, pp. 5127-5131. 